$11-2019$

\title{
Recent updates in the management of merkel cell carcinoma
}

Ahmed Nadeem Abbasi

Aga Khan University, nadeem.abbasi@aku.edu

Syed Mustajab Ahmed

Aga Khan University

Bilal Mazhar Qureshi

Aga Khan University, bilal.qureshi@aku.edu

Follow this and additional works at: https://ecommons.aku.edu/pakistan_fhs_mc_radiat_oncol

Part of the Oncology Commons, and the Radiation Medicine Commons

\section{Recommended Citation}

Abbasi, A. N., Ahmed, S. M., Qureshi, B. M. (2019). Recent updates in the management of merkel cell carcinoma. JPMA. The Journal of the Pakistan Medical Association, 69(11), 1693-1699.

Available at: https://ecommons.aku.edu/pakistan_fhs_mc_radiat_oncol/77 


\title{
Recent updates in the management of Merkel Cell Carcinoma
}

\author{
Ahmed Nadeem Abbasi, Syed Mustajab Ahmed, Bilal Mazhar Qureshi
}

\begin{abstract}
Merkel cell carcinoma is an aggressive nonmelanomatous cutaneous tumour of neuroendocrine origin with an increasing incidence in the recent years. It is a tumour of the elderly and immunosuppressed, which most often appears on sun-exposed areas of the body. The clinical features of the cutaneous or subcutaneous lesions hardly contribute to the diagnosis, and, hence, histopathology and immunohistochemistry play a vital role in diagnosis. The latest staging system by the American Joint Committee on Cancer includes non-nodal invasion to adjacent structures i.e. bone, muscle, fascia, or cartilage into the criteria, in addition to size and depth of invasion. The management relies heavily on a multidisciplinary approach due to rarity of incidence of this disease. According to the international guidelines, surgical management is still the preferred choice. The beneficial role of adjuvant radiotherapy has now been more clearly documented. Data is insufficient to assess whether chemotherapy improves disease-free or overall survival.
\end{abstract}

Keywords: Carcinoma, Merkel cell, Radiotherapy, Keratin20, Keratinocytes. doi: 10.5455/JPMA.286585.

\section{Introduction}

Merkel cell carcinoma (MCC) is an aggressive nonmelanomatous cutaneous tumour of neuroendocrine origin. It was first described in $1972^{1}$ in a study which observed that the tumour originates from the neuroendocrine cells of the basal epidermis of the skin. Later on, studies described the cell of the origin as epidermal, non-dendritic, non-keratinocytic cell that he referred to as a tactile cell. ${ }^{1}$ A number of other terms have also been used to describe this pathology, such as primary small cell carcinoma of the skin, trabecular cell carcinoma, amine precursor uptake decarboxylase (APUDoma) of the skin, and anaplastic cancer of the skin. Being a rare entity, the information pertaining to clinical diagnosis, management, and prognosis of MCC is still in the pipeline. ${ }^{2}$ This review is in series with previous Department of Oncology, Aga Khan University Hospital, Karachi, Pakistan. Correspondence: Syed Mustajab Ahmed. e-mail: smustajabahmed@gmail.com reviews 1,2 providing latest updates on staging and management of MCC.

\section{Incidence and Demographics}

Although MCC is a rare tumour, its incidence has demonstrated an increasing trend in the past two decades. The annual incidence of MCC in the United States is 0.6 per 100,000 . Studies from Australia and New Zealand have documented higher incidence rates. ${ }^{3}$ The average annual incidence between 2006 and 2010, as reported by the Queensland cancer registry data, is 1.6 per 100,000, with a peak rate of 20.7 per 100,000 for individuals 80 years or older. 4 In addition, the annual incidence of MCC recorded in the Netherlands increased from 1.7 in 1993-97 to 3.5 in 2003-07. Some of the factors which have been implicated to be the reason behind the rise of incidence of MCC are increased awareness and improved diagnostic techniques, especially the introduction of cytokeratin 20 (CK20) immuno-staining. The median age at diagnosis in women is 76.2 years whereas that for men is 73.6 years. The incidence is rarer in younger age groups. The incidence of MCC is approximately 5 -fold to 10 -fold greater for people with a solid organ transplant and 11-fold to 13-fold greater in patients with acquired immunodeficiency syndrome (AIDS), suggesting a role of immunosuppression in the pathophysiology of MCC. The National Cancer Database (NCDB) reports that the majority of $\mathrm{MCC}$ cases present with local disease (66\%), followed by nodal disease (27\%), whereas metastatic disease is an even rarer presentation (7\%). Relative survival among patients with local disease was $64 \%$ at five years compared to $39 \%$ in regional nodal disease and $18 \%$ in metastatic disease. ${ }^{3}$

\section{Aetiology}

Although a clear aetiology behind the occurrence of MCC has not been defined yet, it does seem to share natural history, clinical features and behaviour (e.g. high recurrence rate and early spread to regional nodes) with melanoma. ${ }^{1} \mathrm{MCC}$ is a tumour of the elderly and the immunosuppressed, which most often appears on sunexposed areas of the body. In addition to 
immunosuppression and ultraviolet (UV) light exposure, studies have been conducted focussing on the carcinogenesis of MCC, in particular the role of Merkel cell polyomavirus (MCPyV) and its surrogate marker large T-antigen. Together with CK20, other biomarkers, like human insulin gene enhancer-binding protein islet-1 (ISL1) and octamer-binding transcription factor 4 (OCT4) may provide improved methods for diagnosis and ultimately therapy. ${ }^{5}$ The most common site of the primary lesion is head and neck, comprising roughly $50 \%$ of cases (5) and other common sites are extremities (40\%) and trunk (8\%).1,6

\section{Diagnosis}

The usual presentation of MCC is a painless, indurated, solitary dermal nodule with a slightly erythematous to deeply violaceous colour, and, less frequently, an ulcer. In addition, the ability to infiltrate dermal lymphatics, leading to multiple satellite lesions, is also a feature of these lesions. However, the clinical features of the cutaneous or subcutaneous lesions hardly contribute to the diagnosis, and, hence, it is rarely suspected before biopsy. ${ }^{7}$ The clinical features of MCC can be summarised with a mnemonic asymptomatic, expanding rapidly, immunosuppressed, older than 50 years, UV-exposed (AEIOU) skin. ${ }^{8}$ The initial workup for MCC includes ultrasound of the loco-regional lymph nodes and total body scanning examinations. ${ }^{7}$ Histopathology and an incisional or excisional biopsy play a mandatory role in making the diagnosis.

\section{Histopathology}

The tumour is composed of strands or nests of monotonously uniform round blue cells, containing large basophilic nuclei with powdery dispersed chromatin and inconspicuous nucleoli, and minimal cytoplasm. ${ }^{4}$ Other features may include single-cell necrosis, frequent mitoses, lymphovascular invasion, perineural invasion, and epidermal involvement via pagetoid spread, which can be further supported by immunohistochemical (IHC) staining. ${ }^{7}$

\section{Immunohistochemistry}

On IHC examination, Merkel cells show features of both epithelial and neuroendocrine cells. 4 On Immunohistochemical staining (IHC), they express epithelial markers, such as cytokeratin AE1/AE3, CAM

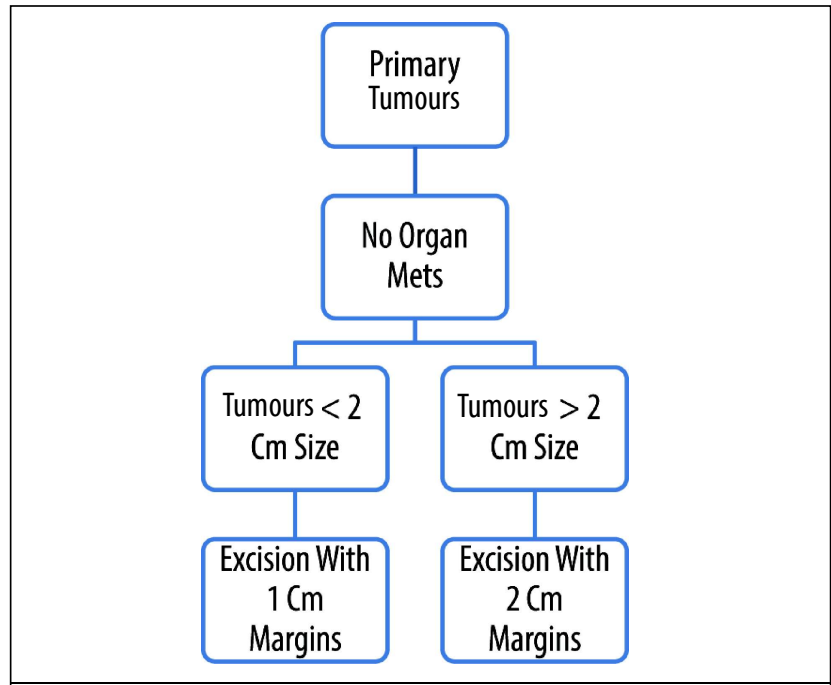

Figure-1 (A): Summarising the key points in incorporating a multidisciplinary approach towards management of Merkel Cell Carcinoma.

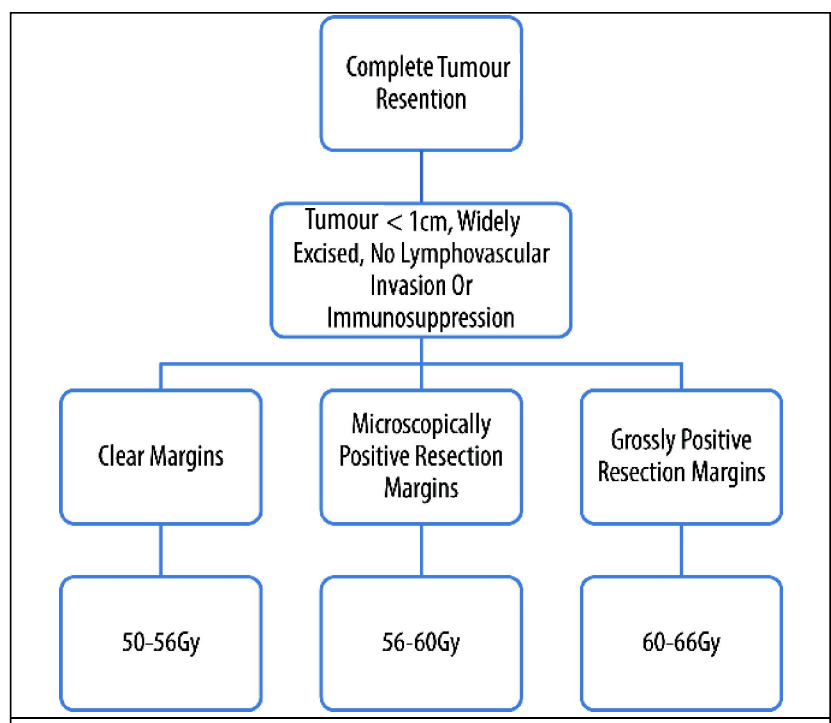

Figure-1 (B): Summarising the key points in incorporating a multidisciplinary approach towards management of Merkel Cell Carcinoma.

5.2, pan-cytokeratin, epithelial membrane antigen, and Ber-EP4, and may stain for various neuroendocrine markers, including chromogranin, synaptophysin, somatostatin, calcitonin, and vasoactive intestinal peptide.

The classic IHC feature of MCC which distinguishes it from other undifferentiated tumours is the immunoreactivity for low-molecular-weight cytokeratins (e.g., CK20, CK5/6). ${ }^{4}$ MCC consistently stains positively for low-molecular-weight CK20, which is a fairly specific and sensitive marker for MCC, with a characteristic paranuclear dot-like positivity. 4 


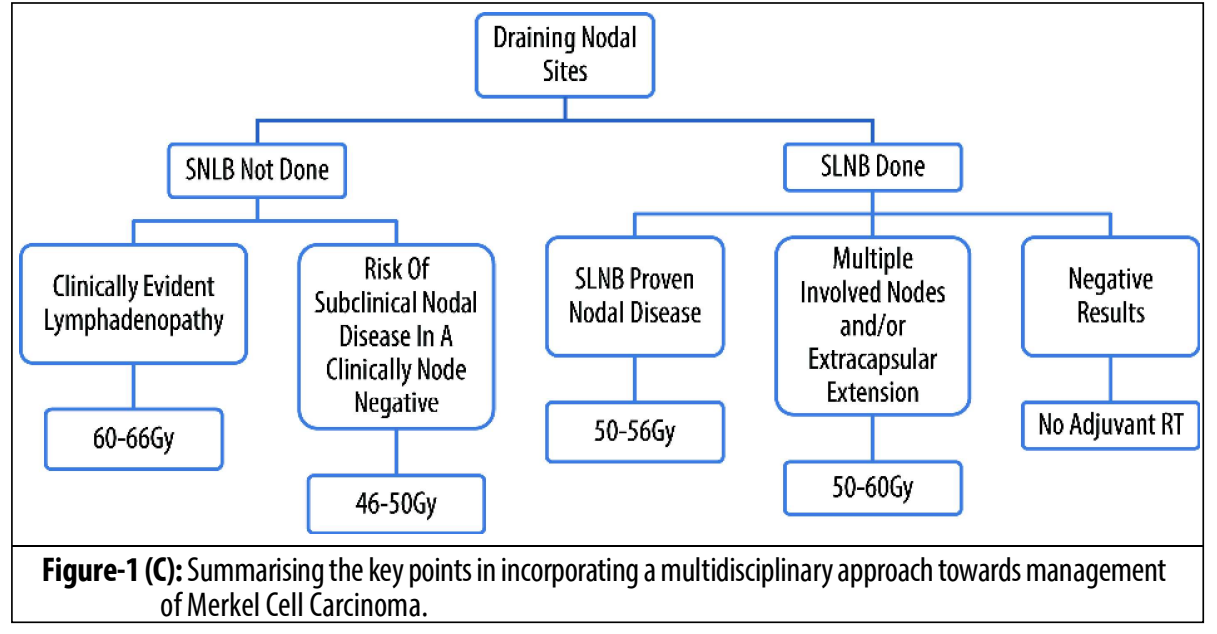

have worse survival compared to those who are staged after pathological examination e.g. Sentinel lymph node biopsy (SLNB). ${ }^{10,12}$

The staging and prognosis of MCC has now been further updated in the eighth edition of the tumour, node, metastasis (TNM) staging system, which has been recommended by both the AJCC and the UICC. ${ }^{13}$ This system has been developed after an analysis of 9387 patients with MCC from the NCDB who were dit provides more detailed correlation with clinical outcomes. The eighth edition provides separate criteria for clinical and pathological staging in contrast to the former staging system. Based upon the TNM information, patients are assigned to prognostic stage groups. These can be summarised as follows:

Stage I: Primary tumours $\leq 2 \mathrm{~cm}$ maximum tumour dimension, without evidence of regional lymph node involvement (Table-1).14,15

Stage II: Primary tumours $>2 \mathrm{~cm}$ (T2 or T3) or a primary tumour with invasion into bone, muscle, fascia, or cartilage (T4), without evidence of lymph node involvement. Stage II is divided into two subgroups based upon the size and depth of invasion of the primary tumour.

Stage III: Any primary tumour with regional lymph node disease. Pathological stage III is divided into subgroups based upon the extent of regional lymph node involvement (Table 2). ${ }^{14,15}$

Stage IV: Metastasis beyond the regional lymph nodes regardless of the status of the primary tumour and regional nodes (Tables 3-4).14,15

Table-1: Tumour, node, metastasis (TNM) staging with respect to size of primary lesion of Merkel Cell Carcinoma according to American Joint Committee on Cancer (AJCC) 8th edition Staging System. $(14,15)$.

\begin{tabular}{ll}
\hline \multicolumn{2}{l}{ Primary Tumour (T) } \\
\hline TX & Primary tumour cannot be assessed (e.g., curetted) \\
T0 & No evidence of primary tumour \\
Tis & In situ primary tumour \\
T1 & Maximum clinical tumour diameter less than or equal to $2 \mathrm{~cm}$ \\
T2 & Maximum clinical tumour diameter $>2$ but less than or equal to $5 \mathrm{~cm}$ \\
T3 & Maximum clinical tumour diameter $>5 \mathrm{~cm}$ \\
T4 & Primary tumour invades fascia, muscle, cartilage, or bone \\
\hline
\end{tabular}


Table-2: Tumour, node and metastasis (TNM) staging with respect to clinical and pathological nodal status of Merkel Cell Carcinoma according to American Joint Committee on Cancer (AJCC) 8th edition Staging System. $(14,15)$.

\begin{tabular}{|c|c|}
\hline \multicolumn{2}{|c|}{ Regional Lymph Nodes (N) Clinical (N) } \\
\hline NX & $\begin{array}{l}\text { Regional lymph nodes cannot be clinically assessed (e.g., previously removed } \\
\text { for another reason, or because of body habitus) }\end{array}$ \\
\hline NO & $\begin{array}{l}\text { No regional lymph node metastasis detected on clinical and/or radiologic } \\
\text { examination }\end{array}$ \\
\hline N1 & Metastasis in regional lymph node(s) \\
\hline N2 & $\begin{array}{l}\text { In-transit metastasis (discontinuous from primary tumour; located between } \\
\text { primary tumour and draining regional nodal basin, or distal to the primary } \\
\text { tumour) without lymph node metastasis }\end{array}$ \\
\hline \multicolumn{2}{|r|}{ Pathological (pN) } \\
\hline pNX & $\begin{array}{l}\text { Regional lymph nodes cannot be assessed (e.g., previously removed for another } \\
\text { reason or not removed for pathological evaluation) }\end{array}$ \\
\hline pNO & No regional lymph node metastasis detected on pathological evaluation \\
\hline pN1 & Metastasis in regional lymph node(s) \\
\hline pN1a(sn) & $\begin{array}{l}\text { ) Clinically occult regional lymph node metastasis identified only by sentinel } \\
\text { lymph node biopsy }\end{array}$ \\
\hline pN1a & Clinically occult regional lymph node metastasis following lymph node dissection \\
\hline pN1b & $\begin{array}{l}\text { Clinically and/or radiologically detected regional lymph node metastasis } \\
\text { microscopically confirmed }\end{array}$ \\
\hline pN2 & $\begin{array}{l}\text { In-transit metastasis (discontinuous from primary tumor; located between } \\
\text { primary tumour and draining regional nodal basin, or distal to the primary } \\
\text { tumour) without lymph node metastasis }\end{array}$ \\
\hline pN3 & $\begin{array}{l}\text { In-transit metastasis (discontinuous from primary tumour; located between } \\
\text { primary tumour and draining regional nodal basin, or distal to the primary } \\
\text { tumour) with lymph node metastasis }\end{array}$ \\
\hline
\end{tabular}

Table-3: Tumour, node, metastasis (TNM) staging with respect to clinical and pathological metastases of Merkel Cell Carcinoma according to American Joint Committee on Cancer (AJCC) 8th edition Staging System. $(14,15)$.

\begin{tabular}{ll}
\hline Distant & Metastasis (M) Clinical (M) \\
\hline M0 & No distant metastasis detected on clinical and/or radiologic examination \\
M1 & Distant metastasis detected on clinical and/or radiologic examination \\
M1a & Metastasis to distant skin, distant subcutaneous tissue, or distant lymph node(s) \\
M1b & Metastasis to lung \\
M1c & Metastasis to all other visceral sites \\
Pathological (M) \\
M0 & No distant metastasis detected on clinical and/or radiologic examination \\
pM1 & Distant metastasis microscopically confirmed \\
pM1a & Metastasis to distant skin, distant subcutaneous tissue, or distant lymph node(s), \\
& microscopically confirmed \\
pM1b & Metastasis to lung, microscopically confirmed \\
pM1c & Metastasis to all other distant sites, microscopically confirmed \\
\hline
\end{tabular}

\section{Management}

MCC is a rare tumour and due to its low incidence and the subtlety of its presentation, its management involves different modalities of oncological treatment. Like many other malignancies, the management of MCC relies mainly on a multidisciplinary approach.16-18 After necessary workup, including histopathological diagnosis
Table-4: Tumour, node, metastasis (TNM) staging of Merkel Cell Carcinoma according to American Joint Committee on Cancer (AJCC) 8th edition Staging System. $(14,15)$.

\begin{tabular}{llll}
\hline Clinical (cTNM) & & & \\
\hline Tis & N0 & M0 & 0 \\
T1 & N0 & M0 & I \\
T2-3 & N0 & M0 & IIA \\
T4 & N0 & M0 & IIB \\
T0-4 & N1-3 & M0 & III \\
T0-4 & Any N & M1 & IV \\
Pathological (pTNM) & & & \\
Tis & N0 & M0 & 0 \\
T1 & N0 & M0 & I \\
T2-3 & N0 & M0 & IIA \\
T4 & N0 & M0 & IIB \\
T1-4 & N1a(sn) or N1a & M0 & IIIA \\
T0 & N1b & M0 & IIIA \\
T1-4 & N1b-3 & M0 & IIIB \\
T0-4 & Any N & M1 & IV \\
\hline
\end{tabular}

and relevant staging workup, each case should be discussed in a multidisciplinary tumour board to reach a decision on further management. ${ }^{16}$ The current recommendations are in favour of surgical intervention being the main course of treatment, 19 but the scarcity of prospective trials investigating this modality is a major factor contributing to the variability of opinion among clinicians. The guidelines for the management of MCC, therefore, explore all approaches i.e. surgery, chemotherapy and radiotherapy (RT).

\section{Surgery}

In order to address the problem of high risk of recurrence of MCC, it has been recommended that the entire lesion be excised at the time of initial presentation to achieve clear surgical margins whenever feasible, keeping in mind that any planned adjuvant RT should not get significantly delayed. The clinical size of the primary lesion plays an important role in the management. For primary tumours without evidence of organ metastases, excision with $1 \mathrm{~cm}$ margins for tumours $<2 \mathrm{~cm}$ in size and $2 \mathrm{~cm}$ margins for those $>2 \mathrm{~cm}$ in size has been recommended (Figure 1-A). ${ }^{20}$ The size of the safety margins may need to be decreased in cases with head and neck involvement to increase aesthetic and functional outcomes. ${ }^{18,21}$ In terms of surgical approach, Mohs micrographic surgery (MMS) has been widely used as a treatment for MCC and has been shown to be as effective as wide local excision (WLE) in treating localised MCC, 22 although the need for concurrent sentinel node 
mapping (and/or neck dissection) suggests that WLE of the primary tumour in the same setting could be a better choice in certain circumstances. ${ }^{20} \mathrm{~A}$ coordinated surgical management is warranted in all cases, so that sentinel lymph node biopsy could be performed prior to definitive surgical intervention, as lymphatic drainage is prone to be altered otherwise. ${ }^{14,15}$ The role of SLNB in the management of MCC has been reflected in a review of 161 MCC patients which found that SLNB identified micro-metastases in one-third of patients. ${ }^{23}$ It has been documented by National Comprehensive Cancer Network (NCCN) panel that identifying patients with nodal disease and subsequently performing nodal dissection and/or RT, enhances the regional control.7,14 However, it should be kept in consideration that SLNB may be less reliable in head and neck compared to trunk and body. 14,15

After verification of clear margins and performing SLNB, if indicated, reconstruction should be performed as soon as possible after surgery. Maximising the efforts to minimise the extensive tissue movement in reconstruction and the delay in adjuvant radiation (if planned) is related to better outcomes. ${ }^{14,15}$

\section{Radiation Treatment}

The rationale of offering RT in the management of MCC has been under debate for a long time. There is relatively little documentation available identifying patients with MCC who have received RT in adjuvant setting (less than 200 published). A study reviewed the Memorial SloanKettering Cancer Centre's MCC database and identified 251 patients who had been treated between 1970 and 2002.24 It analysed patient, tumour, and treatment-related factors for their association with recurrence and survival, but no association was found between irradiation and loco-regional control. 25

The weight of evidence now is in favour of considering adjuvant RT for patients with MCC. 20,26-28 Adjuvant RT has been advocated in order to control local as well as regional disease. ${ }^{26-28} \mathrm{~A}$ systemic review suggests that definitive RT for loco-regional macroscopic MCC provides clinically meaningful local and regional control.29 A metaanalysis comparing the role of surgery alone versus surgery with adjuvant RT demonstrated that the use of adjuvant RT significantly reduced the risk of local and regional recurrence. Adjuvant RT might be considered in patients with multiple affected lymph nodes of extracapsular extension.7
After complete resection of the tumour, the primary disease site must be observed if the tumour is small $(<1 \mathrm{~cm})$, widely excised, and without other risk factors i.e. Iymphovascular invasion or immunosuppression. Radiation-induced toxicity should be considered and discussed with the patient. According to the guidelines formulated by the National Comprehensive Cancer Network, a dose of 50-56Gy should be delivered if the resected margins are clear of the disease, whereas in cases with microscopically positive resection margins, 56-60Gy should be delivered. In case of grossly positive resection margins, or failure to undergo surgery due to unresectability, patient refusal, or significant morbidity, a dose of $60-66 \mathrm{~Gy}$ should be delivered to the primary site (Figure 1-B). ${ }^{14,15}$

Regarding the draining nodal sites, 60-66Gy is recommended for clinically evident lymphadenopathy if the patient has not undergone SLNB or lymph node (LN) dissection, whereas 46-50Gy should be delivered if there is risk of subclinical nodal disease in a clinically node-negative patient in the same setting. For patients who undergo SLNB with negative results, adjuvant RT after surgery is not recommended. However, 50-56Gy should be delivered for SLNB-proven nodal disease. In cases of multiple nodes and/or extracapsular extension, 50-60Gy should be administered after LN dissection (Figure 1-C). ${ }^{14,15}$

RT has also been under consideration in the management of in-transit metastases (stage IIIB) of MCC as it is not possible to contain the disease and ensure clear margins with surgical approach only. ${ }^{30,31}$ Hence, the role of RT has acquired vital importance in this regard. RT to the primary site and satellite lesions with a $3-5 \mathrm{~cm}$ margin with 50Gy in 25 fractions, 31 avoiding interruptions has been recommended, with additional boost to the gross disease (10-16Gy) using electrons.30 This recommendation can be supported by literature that RT can achieve more than $75 \%$ in-field control rates. ${ }^{32}$ Compared to surgery, dermal lypmhatics canbe widely covered using RT. In addition, RT poses fewer late effects than surgery-plus-RT, especially the risk of lymphoedema. 32

Previously, RT has been shown to improve survival in tumours of all sizes, but in current literature, the greatest impact on survival has been in cases where tumours were $>2 \mathrm{~cm} .{ }^{20}$ Data is conflicting as to whether there is any survival benefit from adjuvant primary site or

Vol. 69, No. 11, November 2019 
regional nodal irradiation, partly due to the lack of prospective clinical trials. ${ }^{33}$ The only randomised control trial to date comparing excision with RT versus excision with observation showed no overall survival improvement with adjuvant RT, but showed a significant reduction in regional recurrence. ${ }^{6}$

\section{Chemotherapy}

The effect of adjuvant radiation on survival is currently unproven, but the benefit of chemotherapy seems to be more clearly lacking and should not be recommended routinely. ${ }^{33}$ Chemotherapy is usually reserved for systemic disease, though the success of this treatment is limited, and no chemotherapy protocol has been shown to improve survival. ${ }^{20}$ Although chemotherapy has been used with or without surgery and/or RT for stage IV i.e. cases with distant metastases (M1), it is also being considered for selected cases of macroscopic regional disease ( $\mathrm{N} 1 \mathrm{~b}$ or $\mathrm{N} 2$ ). For local disease, adjuvant chemotherapy is not recommended. 14,15

Nonetheless, data is insufficient to assess whether chemotherapy improves disease-free or overall survival in MCC patients with distant metastases. The most commonly used regimen used is cisplatin or carboplatin with or without etoposide. Topotecan is also considered in some cases of older patients. A regimen comprising cyclophosphamide, doxorubicin and vincristine is commonly considered, but it is associated with significant toxicity. Despite what has been stated, clinicians should exercise evidence-based and patient-centred judgment in choosing chemotherapeutic regimen.14,15

\section{Hyper-thermic isolated limb perfusion}

Although not included in practice guidelines, hyperthermic isolated limb perfusion has been shown to confer some clinical benefits in the management of MCC. A retrospective review suggests that regional perfusion is safe and has a high complete response rate in a selected group of patients, providing durable locoregional control of the disease. ${ }^{34} \mathrm{~A}$ multicentre study evaluating the efficacy of isolated limb perfusion in which combination therapy with melphalan and tumour necrosis factor (TNF) was utilised demonstrated an overall response rate (ORR) of $87.5 \%$ with a complete response (CR) rate of $62.5 \%$. Median loco-regional progressionfree survival (LPFS) was 5 months and median overall survival was 54 months. 35

\section{Conclusion}

Putting all facts in the equation, features which are important in the management of MCC are the rarity of incidence, nonspecific clinical history, and aggressive nature leading to early loco-regional spread, distant metastases and high relapse rates. These features together make MCC a challenge for the team of treating clinicians. Consequently, it is imperative that each case be discussed in a multidisciplinary expert-panel tumour board before embarking on the first treatment modality. The deficiency of literature on MCC to help construct evidence-based management guidelines for clinicians warrants the need for conducting prospective clinical trials.

\section{Disclaimer: None. \\ Conflict Of Interest: None. Source of Funding: None.}

\section{References}

1. Abbasi AN, Zahid S. Latest update on the clinical features and management of Merkel cell carcinoma. J Pak Med Assoc 2012; 62: 262-8.

2. Abbasi AN. Clinical features and management of Merkel cell carcinoma. J Pak Med Assoc 2007; 57: 368-71.

3. Hughes MP, Hardee ME, Cornelius LA, Hutchins LF, Becker JC, Gao L. Merkel Cell Carcinoma: Epidemiology, Target, and Therapy. Curr Dermatol Rep 2014; 3: 46-53.

4. Tai P. Pathogenesis, clinical features, and diagnosis of Merkel cell (neuroendocrine) carcinoma. [Online] 2017 [Cited 2017 December 12]. Available from: URL: https://www.uptodate.com/contents/ pathogenesis-clinical-features-and-diagnosis-of-merkel-cellneuroendocrine-carcinoma\#H9.

5. Huber GF. Modern management of Merkel cell carcinoma. Curr Opin Otolaryngol Head Neck Surg 2014; 22: 109-15.

6. Jouary T, Leyral C, Dreno B, Doussau A, Sassolas B, Beylot-Barry M, et al. Adjuvant prophylactic regional radiotherapy versus observation in stage I Merkel cell carcinoma: a multicentric prospective randomized study. Ann Oncol 2012; 23: 1074-80.

7. Lebbe C, Becker JC, Grob JJ, Malvehy J, Del Marmol V, Pehamberger $\mathrm{H}$, et al. Diagnosis and treatment of Merkel Cell Carcinoma. European consensus-based interdisciplinary guideline. Eur J Cancer 2015; 51: 2396-403.

8. Heath M, Jaimes N, Lemos B, Mostaghimi A, Wang LC, Penas PF, et al. Clinical characteristics of Merkel cell carcinoma at diagnosis in 195 patients: the AEIOU features. J Am Acad Dermatol 2008; 58: 375-81.

9. Yiengpruksawan A, Coit DG, Thaler HT, Urmacher C, Knapper WK. Merkel cell carcinoma. Prognosis and management. Arch Surg 1991; 126: 1514-9.

10. Lemos BD, Storer BE, lyer JG, Phillips JL, Bichakjian CK, Fang LC, et al. Pathologic nodal evaluation improves prognostic accuracy in Merkel cell carcinoma: analysis of 5823 cases as the basis of the first consensus staging system. J Am Acad Dermatol 2010; 63: 75161. 
11. Gupta SG, Wang LC, Penas PF, Gellenthin M, Lee SJ, Nghiem P. Sentinel lymph node biopsy for evaluation and treatment of patients with Merkel cell carcinoma: The Dana-Farber experience and meta-analysis of the literature. Arch Dermatol 2006; 142: 68590.

12. Moshiri AS, Nghiem P. Milestones in the staging, classification, and biology of Merkel cell carcinoma. J Natl Compr Canc Netw 2014; 12: $1255-62$.

13. Tai P. Staging and treatment of Merkel cell carcinoma. [Online] 2017 [Cited 2017 December 12]. Available from: URL: https://www.uptodate.com/contents/staging-and-treatment-ofmerkel-cell-carcinoma.

14. National Comprehensive Cancer N. NCCN Guidelines Version 1.2018 Merkel Cell Carcinoma: National comprehensive cancer, network; 2017. [Online] [Cited 2017 December 12]. Available from: URL: https://www.nccn.org/professionals/physician_gls/pdf/mcc.pdf.

15. Amin MB, Greene FL, Edge SB, Compton CC, Gershenwald JE, Brookland RK, et al. The Eighth Edition AJCC Cancer Staging Manual: Continuing to build a bridge from a population-based to a more "personalized" approach to cancer staging. CA Cancer J Clin 2017; 67: 93-9.

16. Schneider S, Thurnher D, Erovic BM. Merkel cell carcinoma: interdisciplinary management of a rare disease. J Skin Cancer 2013. 2013: 189342

17. Prieto I, Perez de la Fuente T, Medina S, Castelo B, Sobrino B, Fortes $\mathrm{JR}$, et al. Merkel cell carcinoma: An algorithm for multidisciplinary management and decision-making. Crit Rev Oncol Hematol 2016; 98: 170-9.

18. Barksdale SK. Advances in Merkel cell carcinoma from a pathologist's perspective. Pathology 2017; 49: 568-74.

19. Wright GP, Holtzman MP. Surgical resection improves median overall survival with marginal improvement in long-term survival when compared with definitive radiotherapy in Merkel cell carcinoma: A propensity score matched analysis of the National Cancer Database. Am J Surg 2018; 215: 384-7.

20. Ellis DL, Davis RS. Evidence-based management of primary and localized Merkel cell carcinoma: a review. Int J Dermatol 2013; 52: 1248-58.

21. Miles BA, Goldenberg D, Education Committee of the American Head and Neck Society (AHNS). Merkel cell carcinoma: Do you know your guidelines? Head Neck 2016; 38: 647-52.

22. Shaikh WR, Sobanko JF, Etzkorn JR, Shin TM, Miller CJ. Utilization patterns and survival outcomes after wide local excision or Mohs micrographic surgery for Merkel cell carcinoma in the United States,
2004-2009. J Am Acad Dermatol 2018; 78: 175-7.

23. Santamaria-Barria JA, Boland GM, Yeap BY, Nardi V, Dias-Santagata D, Cusack JC Jr. Merkel cell carcinoma: 30-year experience from a single institution. Ann Surg Oncol 2013; 20: 1365-73.

24. Allen PJ, Bowne WB, Jaques DP, Brennan MF, Busam K, Coit DG Merkel cell carcinoma: prognosis and treatment of patients from a single institution. J Clin Oncol 2005; 23: 2300-9.

25. Tai PT, Yu E, Winquist E, Hammond A, Stitt L, Tonita J, et al. Chemotherapy in neuroendocrine/Merkel cell carcinoma of the skin: case series and review of 204 cases. J Clin Oncol 2000; 18: 2493-9.

26. Swanson MS, Sinha UK. Diagnosis and management of merkel cell carcinoma of the head and neck: current trends and controversies. Cancers (Basel) 2014; 6: 1256-66.

27. Bishop AJ, Garden AS, Gunn GB, Rosenthal DI, Beadle BM, Fuller $C D$, et al. Merkel cell carcinoma of the head and neck: Favorable outcomes with radiotherapy. Head Neck 2016; 38 Suppl 1: E4528.

28. Hoeller U, Mueller T, Schubert T, Budach V, Ghadjar P, Brenner W, et al. Regional nodal relapse in surgically staged Merkel cell carcinoma. Strahlenther Onkol 2015; 191: 51-8.

29. Gunaratne DA, Howle JR, Veness MJ. Definitive radiotherapy for Merkel cell carcinoma confers clinically meaningful in-field locoregional control: A review and analysis of the literature. J Am Acad Dermatol 2017; 77: 142-8.

30. Tseng YD, Parvathaneni U. Primary Radiation Therapy for Merkel Cell Carcinoma. Int J Radiat Oncol Biol Phys 2018; 100: 14.

31. Rabinowits G, Yoon C, Thakuria M. Consider Surgery, but Not in This Case. Int J Radiat Oncol Biol Phys 2018; 100: 16.

32. Poulsen M. Radiation Therapy Rather Than Surgery for Merkel Cell Carcinoma: The Advantages of Radiation Therapy. Int J Radiat Oncol Biol Phys 2018; 100: 14-5.

33. Prewett SL, Ajithkumar T. Merkel Cell Carcinoma: Current Management and Controversies. Clin Oncol (R Coll Radiol) 2015; 27: 436-44.

34. Thiels CA, Gonzalez AB, Gray RJ, Jakub JW. Isolated limb perfusion in Merkel cell carcinoma offers high rate of complete response and durable local-regional control: Systematic review and institutional experience. J Surg Oncol 2016; 114: 187-92.

35. van Veenendaal LM, Madu MF, Tesselaar MET, Verhoef C, Grunhagen DJ, van Akkooi ACJ. Efficacy of isolated limb perfusion (ILP) in patients with Merkel cell carcinoma (MCC): A multicenter experience. Eur J Surg Oncol 2017; 43: 2157-62. 